Pure and Applied Mathematics Quarterly

Volume 2, Number 3

(Special Issue: In honor of

Robert MacPherson, Part 1 of 3)

779-794, 2006

\title{
Hessenberg Varieties are not Pure Dimensional
}

\author{
Julianna S. Tymoczko \\ Dedicated to Robert MacPherson
}

\begin{abstract}
We study a family of subvarieties of the flag variety defined by certain linear conditions, called Hessenberg varieties. We compare them to Schubert varieties. We prove that some Schubert varieties can be realized as Hessenberg varieties and vice versa. Our proof explicitly identifies these Schubert varieties by their permutation and computes their dimension.

We use this to answer an open question by proving that Hessenberg varieties are not always pure dimensional. We give examples that neither semisimple nor nilpotent Hessenberg varieties need be pure; the latter are connected, non-pure-dimensional Hessenberg varieties. Our methods require us to generalize the definition of Hessenberg varieties.
\end{abstract}

\section{InTRODUCTION: BACKGROUND AND NOTATION}

A flag is a nested collection of vector spaces $V_{1} \subseteq V_{2} \subseteq \cdots \subseteq V_{n}=\mathbb{C}^{n}$, where each $V_{i}$ is $i$-dimensional. The full flag variety is the complex algebraic variety consisting of all flags; it is smooth and compact.

This paper studies two families of subvarieties of the full flag variety: Hessenberg varieties and Schubert varieties. The first family is defined using two parameters: a linear operator $X: \mathbb{C}^{n} \rightarrow \mathbb{C}^{n}$ and a nondecreasing function $h:\{1,2, \ldots, n\} \longrightarrow\{1,2, \ldots, n\}$. We call $h$ a Hessenberg function. The Hessenberg variety associated to $X$ and $h$ is denoted $\mathcal{H}(X, h)$ and defined by

$$
\mathcal{H}(X, h)=\left\{\text { Flags }: X V_{i} \subseteq V_{h(i)} \text { for all } i\right\} .
$$

(This generalizes the original definition of [dMPS], as in Sections 1.3 and 4.)

For instance, if $X$ is arbitrary and $h$ has $h(i)=n$ for all $i$, then $\mathcal{H}(X, h)$ is the full flag variety. More interesting are the Springer fibers, namely the Hessenberg varieties such that $X$ is nilpotent and $h(i)=i$ for each $i$. Springer fibers are

Received January 5, 2006. 
used to construct geometric representations of the symmetric group ([CG] gives a survey). W. Borho and R. MacPherson generalize Springer representations to a class of Hessenberg varieties that blend these two examples: $h$ is a parabolic function, defined in Section 4 , and $X$ is a nilpotent matrix whose Jordan blocks are subordinate to $h$ (see $[\mathrm{BM}]$ ). More Hessenberg varieties are in Section 1.3.

This paper answers an open question about Hessenberg varieties: are they all pure dimensional? The pure-dimensionality of Springer fibers is significant for Springer representations, which arise from permutation actions on top-dimensional cohomology. Until now, the answer was yes in all known cases.

We show two ways in which Hessenberg varieties can fail to be pure dimensional. In Section 3, we give an example in which $X$ is a semisimple operator and $\mathcal{H}(X, h)$ is a disjoint union of smooth subvarieties of $G / B$ of different dimensions. One case of this example came up in calculations that R. MacPherson and I performed while researching [MT]. In Section 2, we show that $\mathcal{H}(X, h)$ need not be pure dimensional even when $X$ is nilpotent. Section 2 gives a family of examples that are connected but (in general) reducible Hessenberg varieties whose components have different dimensions.

To prove that nilpotent Hessenberg varieties are not always pure, we use Schubert varieties. Every invertible matrix $g$ gives a flag $[g]$ whose $i$-dimensional subspace is spanned by the first $i$ columns of $g$. For each permutation $w$, the Schubert variety $Y_{w}$ is the closure of the set $\{[b w]: b$ is upper-triangular $\}$. Schubert varieties are important because they form a basis for the cohomology of the flag variety. Their geometry is a subject of intense scrutiny and is related to the combinatorics of the symmetric group. For instance, whether $Y_{w}$ is singular is determined by substrings of $w$ [BL, Chapters 5 and 8].

We show that certain Schubert varieties can be realized as Hessenberg varieties, and conversely that some Hessenberg varieties are unions of Schubert varieties. To construct these Schubert varieties, we take $X$ to be the highest weight vector, namely $X=E_{1 n}$. Section 2 describes these Hessenberg varieties in terms of their Schubert-variety components.

Most of this paper treats full flags in $G L_{n}(\mathbb{C})$. Section 4 discusses how to generalize these results to other Lie types. Section 5 contains open questions about Hessenberg varieties, including the question of whether every Schubert variety can be realized as a Hessenberg variety.

The author thanks Konstanze Rietsch and John Stembridge for inspiring and helpful conversations.

1.1. Descriptions of the flag variety. This section is primarily an exposition of three classical ways to describe the flag variety, one geometric, one algebraic, and one combinatorial, all three of which will be used in this paper. This section 
also includes small lemmas needed elsewhere. Our motivation when selecting these proofs was diversity of approach.

1.1.1. Geometric description of the flag variety. Our initial definition was a geometric characterization of the variety of full flags in $\mathbb{C}^{n}$. We denote the flag $V_{1} \subseteq \cdots \subseteq V_{n}$ by $V_{\bullet}$.

Throughout this paper, we use a fixed basis $e_{1}, \ldots, e_{n}$ for $\mathbb{C}^{n}$. Each flag can be written explicitly in terms of this basis.

1.1.2. Algebraic description of the flag variety. The flag $V_{\bullet}$ can be realized (nonuniquely) as an invertible matrix $g$ using the rule that the first $i$ columns of $g$ span $V_{i}$. In this case $V_{\bullet}$ is also denoted $[g]$. Let $B$ denote the group of invertible upper-triangular matrices. The flag variety is the quotient $G L_{n} / B$.

The group $G L_{n}$ acts on the flag variety by the rule that if $h$ is in $G L_{n}$ and $[g]$ is in $G L_{n} / B$ then $h \cdot[g]=[h g]$. When this action is restricted to the uppertriangular matrices $B$, it partitions the flag variety into $B$-orbits whose closures are the Schubert varieties $Y_{w}$.

1.1.3. Combinatorial description of the flag variety. The permutation matrices index Schubert varieties and, as flags, are contained in Schubert varieties. We use $w$ to refer both to the permutation matrix and to the permutation on the set $\{1,2, \ldots, n\}$ defined by $w e_{i}=e_{w(i)}$. We denote transpositions by $s_{i j}$ and denote arbitrary permutations by $w$ or $v$.

For each $w$, the Schubert cell $[B w]$ is the interior of the Schubert variety $Y_{w}$. It can be described explicitly using the following subgroup of $B$.

Definition 1.1. Fix a permutation $w$ and let $U_{w}$ be the subgroup of $B$ defined by either one of the following equivalent conditions:

(1) $U_{w}$ is the maximal subgroup of $B$ such that $w^{-1} U_{w} w$ is lower-triangular with ones along the diagonal.

(2) $U_{w}$ consists of all matrices in $B$ with ones along the diagonal and whose $(i, j)$ entry is zero for each pair $i<j$ with $w^{-1}(i)<w^{-1}(j)$.

The next proposition follows from [H, Sections 28.3 and 28.4].

Proposition 1.2. For each permutation $w$, the following hold:

(1) The set $U_{w} w$ consists of the matrices $\left\{w+u: \begin{array}{c}u \text { is nonzero only in entries that are } \\ \text { both above and to the left of a nonzero entry in } w\end{array}\right\}$.

(2) The matrices $U_{w} w$ are a set of distinct coset representatives for the flags in the Schubert cell $[\mathrm{Bw}]$. (See Figure 1.) 


$$
\left(\begin{array}{lll}
a & 1 & 0 \\
1 & 0 & 0 \\
0 & 0 & 1
\end{array}\right) \quad\left(\begin{array}{lll}
a & b & 1 \\
1 & 0 & 0 \\
0 & 1 & 0
\end{array}\right) \quad\left(\begin{array}{lll}
a & b & 1 \\
c & 1 & 0 \\
1 & 0 & 0
\end{array}\right)
$$

Figure 1. Examples of $U_{w} w$ when $n=3(a, b$, and $c$ are free)

For each permutation $w$, let $w=s_{i_{1}, i_{1}+1} s_{i_{2}, i_{2}+1} \cdots s_{i_{k}, i_{k}+1}$ be a factorization with $k$ as small as possible. We call $k$ the length of $w$, denoted $\ell(w)$. The length of $w$ relates the geometric, algebraic, and combinatorial descriptions of $G L_{n} / B$.

Proposition 1.3. For each permutation $w$, the following hold:

$$
\begin{aligned}
\ell(w) & =\operatorname{dim}(\overline{[B w]}) \\
& =\text { the number of nonzero entries (strictly) above the diagonal in } U_{w} \\
& =\text { the number of pairs } i<j \text { such that } w^{-1}(i)>w^{-1}(j) .
\end{aligned}
$$

Each pair $i<j$ that satisfies $w^{-1}(i)>w^{-1}(j)$ is called an inversion for $w$.

The symmetric group is partially ordered by the Bruhat order. The geometric definition is that $v \leq w$ if and only if $[B v] \subseteq \overline{[B w]}$. Combinatorially, we say $v \leq w$ if and only if there is a factorization $w=s_{i_{1}, i_{1}+1} s_{i_{2}, i_{2}+1} \cdots s_{i_{k}, i_{k}+1}$ so that $v$ can be written as the product of a substring of the $s_{i_{j}, i_{j}+1}$.

1.2. Properties of permutations and Schubert cells. Several lemmas that follow from these properties will be used later in this paper. The difficulty of the proofs depends on which characterization of the flag variety is used. (Each of them is a nice exercise for the reader!)

Lemma 1.4. Fix $j<k \leq n$. For each permutation $w$, the following hold:

(1) The permutation $w s_{j, j+1}$ satisfies $\ell\left(w s_{j, j+1}\right)=\ell(w)-1$ if and only if $w(j)>w(j+1)$. Otherwise $\ell\left(w s_{j, j+1}\right)=\ell(w)+1$.

(2) If $w(j)>w(k)$ then $w>w s_{j k}$ in the Bruhat order.

Proof. The first part is classical, proven by noting that the sets of inversions of $w$ and of $w s_{j, j+1}$ differ exactly by $(w(j), w(j+1))$.

To prove the next part, we show the closure of $[B w]$ contains $w s_{j k}$. Let $u_{w(k), w(j)}(a)$ be the upper-triangular matrix with $a$ in position $(w(k), w(j))$, ones on the diagonal, and zeroes elsewhere. Figure 2 is a schematic of $u_{w(k), w(j)}(a) w$.

Denote the flag $\left[u_{w(k), w(j)}(a) w\right]$ by $V_{1} \subseteq V_{2} \subseteq \cdots \subseteq V_{n}$. The first $j-1$ subspaces and last $n-k+1$ subspaces of this flag agree with those of the flag $\left[w s_{j k}\right]$. The 


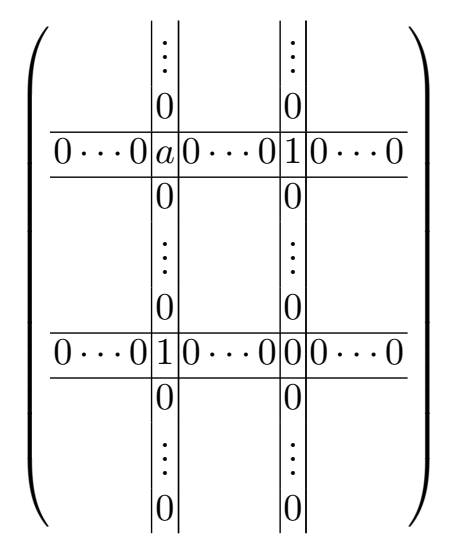

FIGURE 2. Schematic of $u_{w(k), w(j)}(a) w$

other subspaces are

$$
\begin{aligned}
\left\langle V_{j-1},\right. & \left.e_{w(j)}+a e_{w(k)}\right\rangle \subseteq\left\langle V_{j-1}, e_{w(j)}+a e_{w(k)}, e_{w(j+1)}\right\rangle \subseteq \ldots \\
& \subseteq\left\langle V_{j-1}, e_{w(j)}+a e_{w(k)}, e_{w(j+1)}, e_{w(j+2)}, \ldots, e_{w(k-1)}\right\rangle \\
& \subseteq\left\langle V_{j-1}, e_{w(j)}, e_{w(j+1)}, e_{w(j+2)}, \ldots, e_{w(k-1)}, e_{w(k)}\right\rangle=V_{k} .
\end{aligned}
$$

As $a$ approaches $\infty$, these subspaces approach the subspaces

$$
\begin{aligned}
\left\langle V_{j-1},\right. & \left.e_{w(k)}\right\rangle \subseteq\left\langle V_{j-1}, e_{w(k)}, e_{w(j+1)}\right\rangle \subseteq \ldots \\
& \subseteq\left\langle V_{j-1}, e_{w(k)}, e_{w(j+1)}, e_{w(j+2)}, \ldots, e_{w(k-1)}\right\rangle \\
& \subseteq\left\langle V_{j-1}, e_{w(k)}, e_{w(j+1)}, e_{w(j+2)}, \ldots, e_{w(k-1)}, e_{w(j)}\right\rangle=V_{k},
\end{aligned}
$$

which are the corresponding parts of $\left[w s_{j k}\right]$. Thus $\lim _{a \mapsto \infty}\left[u_{w(k), w(j)}(a) w\right]=$ $\left[w s_{j k}\right]$. It follows that $\left[B w s_{j k}\right] \subseteq \overline{[B w]}$, and so $w s_{j k}<w$.

1.3. Hessenberg varieties. In this section, we define Hessenberg varieties algebraically. We also discuss some technical issues that arise.

To obtain an algebraic characterization of Hessenberg varieties, we use subspaces of $n \times n$ matrices rather than the Hessenberg function $h$. The matrix basis unit that is zero except in entry $(i, j)$, where it is one, is denoted $E_{i j}$. Each Hessenberg function defines a subspace of $n \times n$ matrices by $H_{h}=\left\langle E_{i j}: i \leq h(j)\right\rangle$. We call $H_{h}$ a Hessenberg space. The Hessenberg variety of $X$ and $h$ is

$$
\mathcal{H}(X, h)=\left\{\text { Flags }[g]: g^{-1} X g \in H_{h}\right\} .
$$

Many examples of Hessenberg spaces come from classical Lie theory. If $h$ is the Hessenberg function with $h(i)=i$ for each $i$ then $H_{h}$ is the set of upper-triangular matrices. If $h$ is the Hessenberg function given by $h(i)=n$ for each $i$ then $H_{h}$ consists of all $n \times n$ matrices. In fact, if $H_{h}$ is any parabolic subalgebra, then $H_{h}$ 
is a Hessenberg space and the corresponding $h$ is one of the parabolic Hessenberg functions from the Introduction.

Most Hessenberg spaces are not parabolic. For instance, the Hessenberg function given by $h(i)=i+1$ when $i \neq n$ and $h(n)=n$ corresponds to the subspace $H_{h}$ which is zero below the subdiagonal. Figure 3 shows this for $n=4$. Hessenberg

$$
\begin{aligned}
& h(1)=2 \\
& h(2)=3 \\
& h(3)=4 \\
& h(4)=4
\end{aligned} \longleftrightarrow\left(\begin{array}{l}
* * * * \\
* * * * \\
0 * * * \\
00 * *
\end{array}\right)
$$

FIgURE 3. One Hessenberg function and space when $n=4$

varieties with this Hessenberg function are important in various applications, including numerical analysis [dMPS] and computing quantum cohomology of the flag variety (see $[\mathrm{K}]$ and $[\mathrm{R}]$ ).

Our definition of Hessenberg functions omits one condition from the original definition in [dMPS], which also requires $h(i) \geq i$ for each $i$. This paper studies a strictly larger collection of varieties than in [dMPS]. Our generalization is particularly useful when $X$ is nilpotent. (When $X$ is regular semisimple, the variety $\mathcal{H}(X, h)$ will be empty if $h(i)<i$ for each $i$.) Nilpotent Hessenberg varieties arise naturally when studying representations of the symmetric group on Hessenberg varieties that generalize Springer's correspondance [MT].

Section 4 generalizes this definition (and other results) to all Lie types.

Our first proposition establishes that nilpotent Hessenberg varieties depend only on the $i$ for which the Hessenberg function does not satisfy $h(i)=i$.

Proposition 1.5. Fix $n$ and fix $i$ such that $1 \leq i \leq n$. Suppose $h$ is a Hessenberg function with $h(i)=i$ and that the function $h^{\prime}$ defined by

$$
h^{\prime}(j)=\left\{\begin{array}{l}
h(j) \text { if } j \neq i, \text { and } \\
i-1 \text { for } i=j
\end{array}\right.
$$

is also a Hessenberg function. If $X$ is nilpotent then $\mathcal{H}(X, h)=\mathcal{H}\left(X, h^{\prime}\right)$.

Proof. If $g^{-1} X g \in H_{h^{\prime}}$ then $g^{-1} X g \in H_{h}$ since $H_{h^{\prime}} \subseteq H_{h}$. Now assume $g^{-1} X g \in$ $H_{h}$. We have

$$
\left(g^{-1} X g\right) e_{i} \in c e_{i}+\left\langle e_{1}, \ldots, e_{i-1}\right\rangle
$$

where $e_{j}$ are the standard basis vectors for $\mathbb{C}^{n}$. Also

$$
\left(g^{-1} X g\right)\left\langle e_{1}, \ldots, e_{i-1}\right\rangle \subseteq\left\langle e_{1}, \ldots, e_{h(i-1)}\right\rangle \subseteq\left\langle e_{1}, \ldots, e_{i-1}\right\rangle,
$$


since $h(i-1)<h(i)=i$. Since $X$ is nilpotent, applying $g^{-1} X g$ to $e_{i}$ sufficiently many (e.g. $n$ ) times should give zero. On the other hand we have

$$
\left(g^{-1} X g\right)^{n} e_{i} \in c^{n} e_{i}+\left\langle e_{1}, \ldots, e_{i-1}\right\rangle .
$$

Therefore $c=0$, and as a consequence $g^{-1} X g$ lies in $H_{h^{\prime}}$.

Comments from K. Rietsch greatly improved this proof. This lemma motivates the following definition, also suggested by K. Rietsch.

Definition 1.6. For each linear operator $X$, the Hessenberg spaces $H$ and $H^{\prime}$ are $X$-equivalent if $\mathcal{H}(X, H)=\mathcal{H}\left(X, H^{\prime}\right)$. In this case, we write $H \sim_{X} H^{\prime}$ and say that $H$ and $H^{\prime}$ are in the same $X$-equivalence class.

$X$-equivalence of Hessenberg functions is defined the same way.

The $X$-equivalence class of Hessenberg spaces (or functions) depends only on the conjugacy class of $X$ since $\mathcal{H}(X, H) \cong \mathcal{H}\left(g^{-1} X g, H\right)$ (see [T, Proposition $2.7])$.

For instance, if $X=0$ then there is only one $X$-equivalence class of Hessenberg spaces. If $X$ is nilpotent, then the Hessenberg function defined by $h(i)=i$ for all $i$ is $X$-equivalent to the function defined by $h^{\prime}(i)=i-1$ for all $i$. Alternatively, the Hessenberg space consisting of all upper-triangular matrices is $X$-equivalent to the space of all strictly upper-triangular matrices. (This fact is used frequently in Springer theory.) We generalize this in the next corollary, whose proof is immediate from Proposition 1.5.

Corollary 1.7. For each nilpotent linear operator $X$, there is a unique minimal element of each $X$-equivalence class of Hessenberg functions (respectively Hessenberg spaces). This minimal element satisfies

- if there exists $i$ such that $h(i)=i$, then $h(i-1)=i$ as well;

- if there exists a matrix $\sum c_{j k} E_{j k}$ in $H_{h}$ and $i$ such that the coefficient $c_{i i} \neq 0$, then $E_{i i}$ and $E_{i-1, i}$ are both in $H_{h}$.

Typically, we assume $H$ and $h$ are minimal in their $X$-equivalence classes.

\section{Geometry and topology of $\mathcal{X}_{h}$}

In this section, we fix $X$ to be the matrix $E_{1 n}$ and study the Hessenberg varieties

$$
\mathcal{X}_{h}=\left\{\text { Flags }[g]: g^{-1} E_{1 n} g \in H_{h}\right\}=\left\{\text { Flags } V_{1} \subseteq \cdots \subseteq V_{n}: E_{1 n} V_{i} \subseteq V_{h(i)}\right\} .
$$

We will show that these Hessenberg varieties are unions of Schubert varieties. Loosely speaking, each Schubert variety comes from one "corner" of the Hessenberg space. We will identify explicitly these Hessenberg varieties, including which 
Schubert varieties arise and their dimensions. We will also show that many of these Hessenberg varieties are not pure-dimensional.

Proposition 2.1. $\mathcal{X}_{h}$ is a union of Schubert varieties $\bigcup Y_{w}$.

Proof. Each flag can be written in row echelon form as $[u w]$ for some invertible upper-triangular $u$ and permutation matrix $w$. The flag $[u w]$ is in $\mathcal{X}_{h}$ if and only if $w^{-1} u^{-1} E_{1 n} u w$ is in $H_{h}$. Direct calculation shows that $u^{-1} E_{1 n} u$ is a nonzero scalar multiple of $E_{1 n}$ for each upper-triangular $u$. Thus, the flag $[u w]$ is in $\mathcal{X}_{h}$ if and only if $[w]$ is in $\mathcal{X}_{h}$.

This means $\mathcal{X}_{h}$ is a union of Schubert cells, say $\mathcal{X}_{h}=\bigcup[B w]$, and so $\mathcal{X}_{h} \subseteq \bigcup Y_{w}$. Since $\mathcal{X}_{h}$ is closed, it also contains the closures $\bigcup \overline{[B w]}=\bigcup Y_{w}$.

In general the variety $\mathcal{H}(X, h)$ is not a union of Schubert cells $[B w]$. In fact, if $g^{-1} X g$ is another element of the conjugacy class of $X$, then typically at most one of $\mathcal{H}(X, h)$ and $\mathcal{H}\left(g^{-1} X g, h\right)$ is a union of cells $[B w]$, even though the two varieties are homeomorphic [T, Proposition 2.7]. For instance, suppose $n=3$ and the Hessenberg function satisfies $h(i)=i$ for each $i$. Each of $\mathcal{H}\left(E_{12}, h\right)$ and $\mathcal{H}\left(E_{13}, h\right)$ is homeomorphic to two copies of $\mathbb{P}^{1}$ glued together at a point. However, the variety $\mathcal{H}\left(E_{13}, h\right)$ is the union of the Schubert varieties $Y_{s_{1}} \cup Y_{s_{2}}$, while $\mathcal{H}\left(E_{12}, h\right)$ is a one-dimensional closed subvariety of $Y_{s_{2} s_{1}}$.

For each $i \neq j$, let $h_{i j}$ be the Hessenberg function defined by

$$
h_{i j}(k)=\left\{\begin{array}{l}
0 \text { if } k<j \text { and } \\
i \text { if } k \geq j
\end{array}\right.
$$

The corresponding Hessenberg space $H_{i j}$ is spanned by the matrix basis units $E_{k l}$ with $k \leq i$ and $l \geq j$. In other words, $H_{i j}$ is the subspace of matrices which are zero outside of the upper-right $i \times(n-j+1)$ rectangle, as in Figure 4. For

$$
\left(\text { i rows }\{\underbrace{\underbrace{* * * *}_{* * * *}}_{\begin{array}{c}
\mathrm{n}-\mathrm{j}+1 \\
\text { columns }
\end{array}}\}\right.
$$

Figure 4. Schematic diagram of $H_{i j}$

example, $H_{n 1}$ consists of all $n \times n$ matrices and $H_{1 n}$ is just the span of $E_{1 n}$. If the sun rises at the far left of the $i^{t h}$ row, travels around the bottom left corner of the matrix, and sets at the bottom of the $j^{\text {th }}$ column, then $H_{i j}$ is the shadow cast by the matrix basis unit $E_{i j}$ during the course of the 'day'. (A. Ottazzi created this image in $[\mathrm{O}]$.) 
Lemma 2.2. For each pair $i \neq j$, let $w$ be the permutation that has $e_{n}$ in column $j, e_{1}$ in column $i$, and the other vectors inserted in decreasing order $\left(e_{n-1}, e_{n-2}\right.$, $\left.\ldots, e_{2}\right)$ in the remaining columns. Then $\mathcal{X}_{H_{i j}}=Y_{w}$.

Proof. The proof has three parts. First, we show that if $s$ is a permutation, then $[s] \in X_{H_{i j}}$ if and only if $s$ has $e_{1}$ somewhere in its first $i$ columns and $e_{n}$ in its last $n-j+1$ columns. For each such $s$, we form the permutation $s^{\prime}$ by moving $e_{1}$ to the $i^{\text {th }}$ column, moving $e_{n}$ to the $j^{\text {th }}$ column, and keeping the other columns in the same order as in $s$. We then show that $s^{\prime} \geq s$. Finally, we show that $w \geq s^{\prime}$.

Suppose the matrix $s$ has $e_{1}$ in its $k^{\text {th }}$ column and $e_{n}$ in its $l^{\text {th }}$ column. Since $s^{-1}=s^{t}$, the matrix $s^{-1}$ has $e_{1}$ in its $k^{\text {th }}$ row. So

$$
s^{-1} E_{1 n} s=E_{k l} \text {. }
$$

This is in $H_{i j}$ if and only if $k \leq i$ and $l \geq j$. We conclude that the flag $[s] \in X_{H_{i j}}$ if and only if $s^{-1} e_{1}=e_{k}$ for $k \leq i$ and $s^{-1} e_{n}=e_{l}$ for $l \geq j$.

The permutation $w$ satisfies this condition so $\mathcal{X}_{H_{i j}} \supseteq Y_{w}$. We now show that for any permutation $s$ of this form, the flag $[s]$ is in the variety $Y_{w}$.

We begin by moving the column with $e_{n}$ to the left or the column with $e_{1}$ to the right, as long as one of those moves is possible. Suppose $l>j$ and either the $(l-1)^{t h}$ column is not $e_{1}$ or it is $e_{1}$ and $l-1 \neq i$. The flag $\left[s s_{l-1, l}\right]$ is also in $\mathcal{X}_{H_{i j}}$. Lemma 1.4 Part 2 showed $s s_{l-1, l}>s$, so the corresponding Schubert varieties satisfy $Y_{s} \subseteq Y_{s_{l-1, l}}$. (When $k<i$ and either the $(k+1)^{t h}$ column is not $e_{n}$ or it is $e_{n}$ but $k+1 \neq j$, use the flag $\left[s s_{k, k+1}\right]$ in a symmetric argument.)

A move of this sort will be impossible exactly when $j<i$ and either

$$
\begin{aligned}
& \text { - } i=k \text { and } l=k+1=i+1 \text { or } \\
& \text { - } l=j \text { and } k=j-1=l-1 .
\end{aligned}
$$

$$
\left(\begin{array}{l|l}
\cdots & e_{1} \\
e_{n} & \ldots
\end{array}\right)
$$

The diagram is a schematic for these cases: the vectors $e_{1}$ and $e_{n}$ are adjacent, and the $i^{\text {th }}$ column is in place (respectively $j^{\text {th }}$ ) while $e_{n}$ is moving to the left (respectively $e_{1}$ to the right). Lemma 1.4 Part 2 shows that the permutation obtained from $s$ by exchanging its $(i+1)^{t h}$ and $(i-1)^{t h}$ columns is greater than $s$ in the Bruhat order (respectively $j-1$ and $j+1$ ).

Once $e_{1}$ is to the right of $e_{n}$, successively multiply $s$ on the right by $s_{k, k+1}$ or $s_{l-1, l}$ to obtain a permutation $s^{\prime}$ with $s^{\prime} \geq s$, so that $s^{\prime}\left(e_{i}\right)=e_{1}$ and $s^{\prime}\left(e_{j}\right)=e_{n}$.

We now prove by induction that $s^{\prime} \leq w$. Assume that the first $t$ columns of $s^{\prime}$ and $w$ agree and the $(t+1)^{t h}$ does not. The $(t+1)^{t h}$ column of $w$ is filled with $e_{w(t+1)}$. Neither $s^{\prime}(t+1)$ nor $w(t+1)$ is in $\{1, n\}$ because $s^{\prime}(t+1) \neq w(t+1)$. Since $w$ and $s^{\prime}$ agree in the first $t$ columns, the column vector $w\left(e_{t+1}\right)$ is none of $e_{s^{\prime}(1)}$, 
$e_{s^{\prime}(2)}, \ldots, e_{s^{\prime}(t)}$, so there is a positive integer $t_{1}$ such that $s^{\prime}\left(e_{t+1+t_{1}}\right)=e_{w(t+1)}$. The permutation $s^{\prime \prime}=s^{\prime} s_{t+1, t+1+t_{1}}$ satisfies $s^{\prime \prime} \geq s^{\prime}$ by Lemma 1.4 Part 2. Since neither $e_{1}$ nor $e_{n}$ moved, $s^{\prime \prime}$ has $s^{\prime \prime}\left(e_{i}\right)=e_{1}$ and $s^{\prime \prime}\left(e_{j}\right)=e_{n}$, and also agrees with $w$ in its first $t+1$ columns. By induction, the claim follows.

The following corollary restates the condition on $w$.

Corollary 2.3. For each pair $i \neq j$, let $w$ be the largest permutation in the Bruhat order that satisfies $w^{-1} E_{1 n} w=E_{i j}$. Then $X_{H_{i j}}=Y_{w}$.

We can factor $w$ explicitly in terms of simple transpositions.

Corollary 2.4. Let $w_{0}$ be the permutation with $w_{0} e_{k}=e_{n-k+1}$ for each $k=$ $1, \ldots, n$. For each pair $i \neq j$, the Hessenberg variety $X_{H_{i j}}=Y_{w}$, where

$$
w=\left\{\begin{array}{l}
w_{0} s_{12} s_{23} \cdots s_{j-1, j} s_{n, n-1} \cdots s_{i+1, i} \quad \text { if } j<i \text { and } \\
w_{0} s_{12} s_{23} \cdots s_{j-2, j-1} s_{n, n-1} \cdots s_{i+1, i} \text { if } j>i .
\end{array}\right.
$$

Proof. For each matrix $M$, the product $M s_{12} s_{23} \cdots s_{k, k+1}$ cyclically permutes the first $k+1$ columns of $M$, sending the first column to the $(k+1)^{t h}$ position and moving each of the other columns one position to the left. Similarly, the product $M s_{n, n-1} s_{n-1, n-2} \cdots s_{k+1, k}$ cyclically permutes the last $n-k+1$ columns, moving the last column to the $k^{\text {th }}$ and moving the others one column to the right. Cyclically permuting the first $j$ (respectively $j-1$ ) columns and the last $n-i+1$ columns of $w_{0}$ gives the permutation $w$ of Lemma 2.2 .

This gives a closed formula for the dimension of $X_{H_{i j}}$.

Corollary 2.5. For each $i \neq j$, the dimension of $X_{H_{i j}}$ is

$$
\left\{\begin{array}{l}
\left(\begin{array}{l}
n \\
2
\end{array}\right)-(j-1+n-i) \text { if } j<i \text { and } \\
\left(\begin{array}{l}
n \\
2
\end{array}\right)-(j-2+n-i) \text { if } j>i .
\end{array}\right.
$$

Proof. The length of the permutation $w_{0}$ is $\left(\begin{array}{l}n \\ 2\end{array}\right)$. Let $w=w_{0} \prod s_{k, k+1}$ be the factorization from Corollary 2.4. Each simple transposition in this factorization reduces the length of $w_{0}$ by one, from Lemma 1.4 Part 1.

2.1. The components of $\mathcal{X}_{H}$. It is usually difficult to identify the irreducible components of Hessenberg varieties. However, when $X=E_{1 n}$, it can be done.

Proposition 2.6. For all $H$ and $H^{\prime}$, we have $\mathcal{X}_{H \cup H^{\prime}}=\mathcal{X}_{H} \cup \mathcal{X}_{H^{\prime}}$. 
Proof. The flag $[w]$ is in $\mathcal{X}_{H \cup H^{\prime}}$ if and only if $w^{-1} E_{1 n} w$ is in $H \cup H^{\prime}$. Since $w^{-1} E_{1 n} w$ is a matrix basis unit, it is in $H \cup H^{\prime}$ if and only if either $w^{-1} E_{1 n} w$ is in $H$ or $w^{-1} E_{1 n} w$ is in $H^{\prime}$. This holds if and only if the flag $[w]$ is in $\mathcal{X}_{H} \cup \mathcal{X}_{H^{\prime}}$.

Lemma 2.7. Let $H$ and $H^{\prime}$ be Hessenberg spaces that are minimal in their $E_{1 n^{-}}$ equivalence classes. Then $\mathcal{X}_{H} \subseteq \mathcal{X}_{H^{\prime}}$ if and only if $H \subseteq H^{\prime}$.

Proof. We reduce to the case when $\mathcal{X}_{H}$ and $\mathcal{X}_{H^{\prime}}$ are Schubert varieties. Write $H=\bigcup H_{i j}$ and $H^{\prime}=\bigcup H_{i^{\prime} j^{\prime}}^{\prime}$. Each $H_{i j}$ satisfies $i \neq j$ since $H$ is minimal in its $E_{1 n}$-equivalence class (respectively $i^{\prime} \neq j^{\prime}$ ). For each pair $i \neq j$, the matrix $E_{i j}$ is in $H^{\prime}$ if and only if $E_{i j} \in H_{i^{\prime} j^{\prime}}^{\prime}$ for some $i^{\prime}, j^{\prime}$. This holds if and only if $H_{i j} \subseteq H_{i^{\prime} j^{\prime}}^{\prime}$. Consequently $H \subseteq H^{\prime}$ if and only if for each $i, j$ there exist $i^{\prime}, j^{\prime}$ such that $H_{i j} \subseteq H_{i^{\prime} j^{\prime}}^{\prime}$. We know $\mathcal{X}_{H}=\bigcup \mathcal{X}_{H_{i j}}$ and $\mathcal{X}_{H^{\prime}}=\bigcup \mathcal{X}_{H_{i^{\prime} j^{\prime}}^{\prime}}$ from Proposition 2.6. It suffices to show that $\mathcal{X}_{H_{i j}} \subseteq \mathcal{X}_{H_{i^{\prime} j^{\prime}}^{\prime}}$ if and only if $H_{i j} \subseteq H_{i^{\prime} j^{\prime}}$.

Both $\mathcal{X}_{H_{i j}}$ and $\mathcal{X}_{H_{i^{\prime} j^{\prime}}}$ are a disjoint union of Schubert cells by Proposition 2.1. This means the inclusion $\mathcal{X}_{H_{i j}} \subseteq \mathcal{X}_{H_{i^{\prime} j^{\prime}}}$ holds if and only if each permutation flag $[s]$ in $\mathcal{X}_{H_{i j}}$ is also contained in $\mathcal{X}_{H_{i^{\prime} j^{\prime}}}$. Equation 1 shows that $[s]$ is in $\mathcal{X}_{H_{i j}}$ if and only if $s^{-1} E_{1 n} s=E_{k l}$, where $k$ and $l$ satisfy the conditions $k \leq i$ and $l \geq j$. It follows that each permutation flag $[s]$ in $\mathcal{X}_{H_{i j}}$ is also in $\mathcal{X}_{H_{i^{\prime} j^{\prime}}}$ if and only if $i \leq i^{\prime}$ and $j \geq j^{\prime}$, which is true if and only if $H_{i j} \subseteq H_{i^{\prime} j^{\prime}}$.

Definition 2.8. A maximal decomposition of the Hessenberg space $H$ is a union $H=\bigcup H_{i j}$ so that no pair $H_{i j}, H_{i^{\prime} j^{\prime}}$ satisfies $H_{i j} \subseteq H_{i^{\prime} j^{\prime}}$.

If $H$ is minimal in its $E_{1 n}$-equivalence class, then a maximal decomposition $H=\bigcup H_{i j}$ further satisfies $i \neq j$ for each $H_{i j}$.

Corollary 2.9. Let $H$ be minimal in its $E_{1 n}$-equivalence class. If $H=\bigcup H_{i j}$ is a maximal decomposition, the components of $\mathcal{X}_{H}$ are the Schubert varieties $\mathcal{X}_{H_{i j}}$.

Proof. Write $\mathcal{X}_{H}=\bigcup \mathcal{X}_{H_{i j}}$ as in Proposition 2.6. For each $H_{i j}$, there is a unique permutation $w_{i j}$ such that $\left[B w_{i j}\right]$ is dense in $\mathcal{X}_{H_{i j}}$ by Lemma 2.2. For every $\left(i^{\prime}, j^{\prime}\right) \neq(i, j)$, Lemma 2.7 shows that $\left[w_{i^{\prime} j^{\prime}}\right]$ is not in $\mathcal{X}_{H_{i j}}$, and so $\left[B w_{i^{\prime} j^{\prime}}\right] \cap \mathcal{X}_{H_{i j}}$ is empty. This means $\mathcal{X}_{H_{i j}}$ is an irreducible component of $\mathcal{X}_{H}$.

Corollary 2.10. Fix $H$, a minimal Hessenberg space in its $E_{1 n}$-equivalence class. The Hessenberg variety $\mathcal{X}_{H}$ is pure dimensional if and only if there exists an integer $k \in\{1,2, \ldots, n-1\}$ and a subset $I \subseteq\{1,2, \ldots, n-k\}$ such that either $H=\bigcup_{i \in I} H_{i, i+k}$ or $H=\bigcup_{i \in I} H_{i, i-k}$.

Proof. Let $H=\bigcup H_{i j}$ be a maximal decomposition and write $\mathcal{X}_{H}$ as a union of its irreducible components $X_{H_{i j}}$. Each $X_{H_{i j}}$ is a Schubert variety that has dimension $\left(\begin{array}{l}n \\ 2\end{array}\right)-(j-1+n-i)$ if $j<i$ and $\left(\begin{array}{l}n \\ 2\end{array}\right)-(j-2+n-i)$ if $j>i$ by 
Corollary 2.5. Given pairs $(i, j)$ and $\left(i^{\prime}, j^{\prime}\right)$, the varieties $X_{H_{i j}}$ and $X_{H_{i^{\prime} j^{\prime}}}$ have the same dimension if and only if $j-i=j^{\prime}-i^{\prime}$.

This gives a collection of examples of nilpotent Hessenberg varieties that are connected and not pure dimensional. For instance, the Hessenberg space $H=$ $H_{41} \cup H_{54}$ of $5 \times 5$ matrices gives a variety $\mathcal{X}_{H}$ in $G L_{5} / B$ that is not pure.

\section{A SEMisimple Hessenberg VARIETy that is NOt PURE Dimensional}

In this section, we describe another way that Hessenberg varieties can fail to be pure dimensional. The next proposition generalizes an example that $\mathrm{R}$. MacPherson and I discovered.

Proposition 3.1. Fix $X=\sum_{i=1}^{n-1} E_{i i}$. Let $h$ be the Hessenberg function with $h(i)=n-1$ for all $i \leq n-1$, and $h(n)=n$. The variety $\mathcal{H}(X, h)$ is the disjoint union of two components, one of which is homeomorphic to $G L_{n-1} / B$ and the other of which is homeomorphic to a fiber bundle over $\mathbb{P}^{n-2}$ with fiber $G L_{n-1} / B$. In particular, the Hessenberg variety $\mathcal{H}(X, h)$ is not pure dimensional.

Proof. By definition, each flag $V_{\bullet}$ in $\mathcal{H}(X, h)$ satisfies $X V_{n-1} \subseteq V_{n-1}$. Since $X\left(\sum_{i=1}^{n} a_{i} e_{i}\right)=\sum_{i=1}^{n-1} a_{i} e_{i}$, either

(1) $e_{n} \in V_{n-1}$ or

(2) $V_{n-1}=\left\langle e_{1}, e_{2}, \ldots, e_{n-1}\right\rangle$.

These conditions are closed and so define two closed subvarieties $\mathcal{Y}_{1}$ and $\mathcal{Y}_{2}$, respectively, in $G L_{n} / B$. The two conditions cannot be simultaneously satisfied so $\mathcal{H}(X, h)$ is the disjoint union $\mathcal{Y}_{1} \cup \mathcal{Y}_{2}$. We now describe these subvarieties.

First we show that $\mathcal{Y}_{2} \cong G L_{n-1} / B$. The flag $[g]$ satisfies Condition 2 if and only if the matrix $g$ is in

$$
P=\left(\begin{array}{c|c} 
& * \\
G L_{n-1} & * \\
& \vdots \\
& * \\
\hline 00 \cdots 0 & \mathbb{C}^{*}
\end{array}\right) .
$$

In other words, the component $\mathcal{Y}_{2}$ is isomorphic to $G L_{n-1} / B$ via the isomorphism that sends $V_{1} \subseteq \cdots \subseteq V_{n-1} \subseteq V_{n}$ to the flag $V_{1} \subseteq \cdots \subseteq V_{n-1}$ inside $\left\langle e_{1}, \ldots, e_{n-1}\right\rangle$.

Now we study $\mathcal{Y}_{1}$. Denote the Grassmannian of $n-1$-planes in $\mathbb{C}^{n}$ by $G(n-1, n)$. Write $\pi_{n-1}: G L_{n} / B \longrightarrow G(n-1, n)$ for the projection that sends the flag $V_{1} \subseteq V_{2} \subseteq \cdots \subseteq V_{n}$ to the subspace $V_{n-1}$. This is a continuous map; in fact, it is the quotient map $\pi_{n-1}: G L_{n} / B \longrightarrow G L_{n} / P$. 
Restrict the map to $\left.\pi_{n-1}\right|_{\mathcal{Y}_{1}}: \mathcal{Y}_{1} \longrightarrow G(n-1, n)$. The image $\pi_{n-1}\left(\mathcal{Y}_{1}\right)$ is

$$
\pi_{n-1}\left(\mathcal{Y}_{1}\right)=\left\{\text { Subspaces } V_{n-1} \text { such that } e_{n} \in V_{n-1}\right\} \text {. }
$$

This is isomorphic to the set of $n-2$-dimensional subspaces in $\left\langle e_{1}, \ldots, e_{n-1}\right\rangle$, so $\pi_{n-1}\left(\mathcal{Y}_{1}\right) \cong G(n-2, n-1)$. Since $G(n-2, n-1) \cong \mathbb{P}^{n-2}$, we conclude that the image $\pi_{n-1}\left(\mathcal{Y}_{1}\right) \cong \mathbb{P}^{n-2}$.

We now identify the fiber $\left(\pi_{n-1} \mid \mathcal{Y}_{1}\right)^{-1}\left(V_{n-1}\right)$ of each $V_{n-1} \in \pi_{n-1}\left(\mathcal{Y}_{1}\right)$. The flag $W_{1} \subseteq \cdots \subseteq W_{n}$ is in $\left(\pi_{n-1} \mid \mathcal{Y}_{1}\right)^{-1}\left(V_{n-1}\right)$ if and only if $W_{n-1}=V_{n-1}$. Every flag in $G L_{n} / B$ satisfies $W_{n}=\mathbb{C}^{n}$, so the fiber is characterized by

$$
\left(\pi_{n-1} \mid \mathcal{Y}_{1}\right)^{-1}\left(V_{n-1}\right)=\left\{\text { Flags such that } W_{1} \subseteq W_{2} \subseteq \cdots W_{n-2} \subseteq V_{n-1}\right\} .
$$

This is the set of complete flags in $V_{n-1}$ and is homeomorphic to $G L_{n-1} / B$.

Consequently, the map $\pi_{n-1}: \mathcal{Y}_{1} \longrightarrow \pi_{n-1}\left(\mathcal{Y}_{1}\right)$ is a fiber bundle whose base space is homeomorphic to $\mathbb{P}^{n-2}$ and whose fiber is homeomorphic to $G L_{n-1} / B$.

For example, when $n=3$ the Hessenberg variety $\mathcal{H}(X, h)$ is a disjoint union of $\mathbb{P}^{1}$ and a $\mathbb{P}^{1}$-bundle over $\mathbb{P}^{1}$.

\section{Generalizing to all Lie types}

In this section, we discuss generalizations of these results to arbitrary Lie type. Our exposition is brief; we assume our reader is familiar with the general theory.

Let $G$ be a complex reductive linear algebraic group, $\mathfrak{g}$ its Lie algebra, $B$ a fixed Borel subgroup, and $\mathfrak{b}$ its Lie algebra. The full flag variety is $G / B$ and its elements are written $[g]$. Let $T$ be a maximal torus contained in $B$ and $\mathfrak{t}$ be the Cartan subalgebra associated to $T$. We will also use $\mathfrak{n}^{-}$, the maximal nilpotent subalgebra in the opposite Borel subalgebra $\mathfrak{b}^{-}$. Let $W$ be the Weyl group.

The positive roots in the root system corresponding to $\mathfrak{g}$ are denoted $\Phi^{+}$and the negative roots are $\Phi^{-}$. The inner product on $\Phi$ is written $\langle\cdot, \cdot\rangle$. We refer to the length of roots, which can be either short or long. If $\alpha$ and $\beta$ are two roots, then $\alpha \succ \beta$ means $\alpha-\beta$ is a sum of positive roots. (Note that this is not the partial ordering where $\alpha>\beta$ means $\alpha-\beta$ is a positive root.) If $\alpha=\sum c_{i} \alpha_{i}$ is a (reduced) sum of simple roots, then the support of $\alpha$ is the set $\operatorname{supp}(\alpha)=\left\{\alpha_{i}: c_{i} \neq 0\right\}$. Given $\alpha$, we write $E_{\alpha}$ for a root vector corresponding to $\alpha$.

A Hessenberg space $H$ is a linear subspace of matrices such that $[H, \mathfrak{b}] \subseteq H$. (This definition omits one condition from that found in [dMPS].) Suppose $X$ is in $\mathfrak{g}$ and $H$ is a Hessenberg space. The Hessenberg variety of $(X, H)$ is given by

$$
\mathcal{H}(X, H)=\left\{[g] \in G / B: g^{-1} X g \in H\right\} .
$$


Proposition 4.1. Let $E_{\theta}$ be a weight vector for the highest weight $\theta$. For each Hessenberg space $H$, the variety $\mathcal{H}\left(E_{\theta}, H\right)$ is a union of Schubert varieties.

This generalizes Proposition 2.1. The proof is the same as in Proposition 2.1: the flag $[b w]$ is in $\mathcal{H}\left(E_{\theta}, H\right)$ if and only if $w^{-1} b^{-1} E_{\theta} b w$ is in $H$, and the adjoint action of $B$ multiplies $E_{\theta}$ by a nonzero constant factor.

Definition 4.2. For each root $\alpha$, define $H_{\alpha}$ to be minimal with respect to inclusion among all Hessenberg spaces that contain the root vector $E_{\alpha}$.

If $\alpha$ is positive then $H_{\alpha}$ is the span of the root vectors $E_{\beta}$ with $\beta \succeq \alpha$. However, this is not true when $\alpha$ is negative. In that case, every positive root $\beta$ satisfies $\beta \succeq \alpha$, but $H_{\alpha}$ need not contain $\mathfrak{b}$.

Let $N(\operatorname{supp}(\alpha))=\left\{\alpha_{j}: \exists \alpha_{i} \in \operatorname{supp}(\alpha)\right.$ with $\left.\left\langle\alpha_{j}, \alpha_{i}\right\rangle \neq 0\right\}$. In other words, $N(\operatorname{supp}(\alpha))$ consists of $\operatorname{supp}(\alpha)$ as well as the simple roots that are joined to a root in $\operatorname{supp}(\alpha)$ by an edge in the Dynkin diagram for $\mathfrak{g}$.

Lemma 4.3. Let $\alpha \in \Phi^{-}$. If $H_{\alpha}^{+}=\left\langle E_{\beta}: \beta \in \Phi^{+}, \operatorname{supp}(\beta) \cap N(\operatorname{supp}(\alpha)) \neq \emptyset\right\rangle$, $H_{\alpha}^{-}=\left\langle E_{\beta}: \beta \in \Phi^{-}\right.$has $\left.\beta \succeq \alpha\right\rangle$, and $T_{\alpha}=\left\langle\left[E_{\alpha_{i}}, E_{-\alpha_{i}}\right]: \alpha_{i} \in \operatorname{supp}(\alpha)\right\rangle$, then

$$
H_{\alpha}=H_{\alpha}^{-} \oplus H_{\alpha}^{+} \oplus T_{\alpha} \text {. }
$$

Proof. Recall that $\left[E_{\beta}, E_{\gamma}\right]$ is a nonzero multiple of $E_{\beta+\gamma}$ if $\beta+\gamma$ is a root, an element $T_{\beta}$ of the Cartan subalgebra if $\gamma=-\beta$, and zero otherwise.

This identity implies that

$$
H_{\alpha}^{-}=\bigcap_{\substack{\text { Hess. spaces } H \\ \text { s.t. } E_{\alpha} \in H}} H \cap \mathfrak{n}^{-}
$$

and that the Cartan subalgebra $\mathfrak{t}$ intersects $\left[H_{\alpha}^{-}, \mathfrak{b}\right]$ exactly in $T_{\alpha}$. The $[\mathfrak{b}, \cdot]$ closure of $T_{\alpha}$ is $H_{\alpha}^{+}$.

We must show that $\left[H_{\alpha}^{-}, \mathfrak{b}\right] \cap \mathfrak{b} \subseteq T_{\alpha} \oplus H_{\alpha}^{+}$. Suppose $E_{\gamma} \in \mathfrak{b}$ and $E_{\beta} \in H_{\alpha}^{-}$ satisfy $\gamma+\beta \in \Phi^{+}$. We will find a simple root $\alpha_{i} \in \operatorname{supp}(\gamma+\beta) \cap N(\operatorname{supp}(\alpha))$. If the support of $\beta$ is contained in the support of $\gamma+\beta$, then any $\alpha_{i} \in \operatorname{supp}(\beta)$ is as desired, $\operatorname{since} \operatorname{supp}(\beta) \subseteq \operatorname{supp}(\alpha)$ by definition of $H_{\alpha}^{-}$. If $\operatorname{supp}(\beta) \nsubseteq \operatorname{supp}(\gamma+\beta)$ then we may write the support of $\gamma$ as the (not necessarily disjoint) union $\operatorname{supp}(\gamma)=\operatorname{supp}(\beta) \cup \operatorname{supp}(\gamma+\beta)$. Suppose for every $\alpha_{i} \in \operatorname{supp}(\gamma+\beta)$ and every $\alpha_{j} \in \operatorname{supp}(\alpha)$ we have $\left\langle\alpha_{i}, \alpha_{j}\right\rangle=0$. Then the support of $\gamma+\beta$ is not connected to the support of $\alpha$ in the Dynkin diagram, and consequently the support of $\gamma+\beta$ is not connected to the support of $\beta$. In other words, the support of $\gamma$ is a disconnected subset of the Dynkin diagram. This contradicts the fact that the support of each root is a connected subset of the Dynkin diagram (see [B, page 169]). So $E_{\gamma+\beta} \in H_{\alpha}^{+}$. 
The next two results generalize Lemma 2.2.

Lemma 4.4. If $\alpha$ is a root of the same length as $\theta$, there is a unique maximal Weyl group element $w$ that satisfies $w^{-1} \theta=\alpha$.

Proof. Denote the stabilizer of $\theta$ in $W$ by $\operatorname{Stab}(\theta)$. Consider the left cosets $\operatorname{Stab}(\theta) \backslash W$. Each coset has a unique maximal element $\operatorname{since} \operatorname{Stab}(\theta)$ is parabolic. Also, each coset $\operatorname{Stab}(\theta) u$ is determined by the root $u^{-1} \theta$. Since $\alpha$ and $\theta$ are in the same $W$-orbit, there exists $w$ with $w^{-1} \theta=\alpha$.

Proposition 4.5. Let $\alpha$ be a root of the same length as $\theta$ and let $w$ be the maximal Weyl group element with $w^{-1} \theta=\alpha$. Then $\mathcal{H}\left(E_{\theta}, H_{\alpha}\right)=Y_{w}$.

Proof. For each element $u$ in $W$, the flag $[u]$ is in $\mathcal{H}\left(E_{\theta}, H_{\alpha}\right)$ if and only if $u^{-1} E_{\theta} u \in H_{\alpha}$. Since $u^{-1} E_{\theta} u=E_{u^{-1} \theta}$, the Hessenberg variety $\mathcal{H}\left(E_{\theta}, H_{\alpha}\right)$ is a union of Schubert cells indexed by the elements in cosets of $\operatorname{Stab}(\theta) \backslash W$. We must show that if $E_{u^{-1} \theta} \in H_{\alpha}$ then $w \geq u$ in the Bruhat order.

The roots $u^{-1} \theta$ and $w^{-1} \theta$ have the same length. If $u^{-1} \theta$ and $w^{-1} \theta$ have the same sign, then $u^{-1} \theta \succeq w^{-1} \theta$ if and only if $w \geq u$ by [St, Proposition 3.2]. Now suppose $u^{-1} \theta$ is positive and $w^{-1} \theta$ is negative. Without loss of generality, let $u^{-1} \theta=\alpha_{i}$ be simple. If $\alpha_{i}$ is in the support of $w^{-1} \theta$ then $s_{i} u^{-1} \theta \succeq w^{-1} \theta$ and so $w \geq u s_{i}$. Moreover, we know $u s_{i}>u$ since $u s_{i} \alpha_{i} \in \Phi^{-}$. This gives $w \geq u$.

If $\alpha_{i}$ is not in the support of $w^{-1} \theta$ then there exists an $\alpha_{j} \in \operatorname{supp}\left(w^{-1} \theta\right)$ such that $\left\langle\alpha_{i}, \alpha_{j}\right\rangle \neq 0$ by Lemma 4.3. At least one simple root in $\operatorname{supp}\left(w^{-1} \theta\right)$ has the same length as $w^{-1} \theta$ and hence as $\alpha_{i}$. If the Dynkin diagram for $\mathfrak{g}$ has a multiedge, then the simple roots are long on one side of the multiedge and short on the other. So the edge from $\alpha_{i}$ to $\alpha_{j}$ cannot be a multiedge. This means that $s_{j} \alpha_{i}=s_{i} \alpha_{j}=\alpha_{i}+\alpha_{j}$. The root $s_{j} u^{-1} \theta=\alpha_{i}+\alpha_{j}$ and so $u>u s_{j}$ by [St, Proposition 3.2]. Let $u=v s_{j}$ be a reduced factorization. Then $s_{j} s_{i} v^{-1} \theta=-\alpha_{j}$ and $w \geq v s_{i} s_{j}$, again by [St, Proposition 3.2]. The factorization $v s_{i} s_{j}$ is reduced because $v s_{i}>v\left(\left[\right.\right.$ St, Proposition 3.2]) and $v s_{i} s_{j}>v s_{i}$ (because $v s_{i} s_{j} \alpha_{j} \in \Phi^{-}$). We conclude that $w \geq v s_{i} s_{j}>u$.

\section{Questions}

We first ask about the relation between Schubert and Hessenberg varieties.

Question 5.1. Are all Schubert varieties Hessenberg varieties? If not, describe explicitly the Schubert varieties that are also Hessenberg varieties.

The matrices in the Hessenberg spaces of Corollary 2.10 are said to be in banded Hessenberg form, a form used in numerical analysis (see [dMPS]). We ask if this algebraic property is related to the geometric condition of purity. 
Question 5.2. Let $X$ be any linear operator. If $H$ is in banded Hessenberg form, is the Hessenberg variety $\mathcal{H}(X, H)$ necessarily pure-dimensional?

The next question arises because of the representations on the cohomology of Springer fibers. We wonder whether the highest-weight Hessenberg varieties of Sections 2 and 4 also carry interesting geometric actions.

Question 5.3. Does the cohomology of the highest-weight Hessenberg varieties carry interesting group actions? Is there an interesting group action on the highest-weight Hessenberg variety that permutes its irreducible components?

[dMPS] proved that regular semisimple Hessenberg varieties are smooth.

Question 5.4. Are all semisimple Hessenberg varieties smooth?

\section{REFERENCES}

[BL] S. Billey and V. Lakshmibai, Singular loci of Schubert varieties, Progress in Math. 182, Birkhauser, Boston, 2000.

[BM] W. Borho and R. MacPherson, Partial resolutions of nilpotent varieties, Analysis and topology on singular spaces, II, III (Luminy, 1981), 23-74, Astérisque 101-102, Soc. Math. France, Paris, 1983.

[B] N. Bourbaki, Groupes et Algèbres de Lie, Chp. IV-VI, Masson, Paris, 1981.

[CG] N. Chriss and V. Ginzburg, Representation theory and complex geometry, Birkhauser, Boston, 1997.

[GKM] M. Goresky, R. Kottwitz, and R. MacPherson, "Equivariant cohomology, Koszul duality, and the localization theorem," Invent. Math. 131 (1998), 25-83.

[H] J. Humphreys, Linear Algebraic Groups, Grad. Texts in Math. 21, Springer-Verlag, New York, 1964.

[K] B. Kostant, "Flag manifold quantum cohomology, the Toda lattice, and the representation with highest weight $\rho$," Selecta Math. (N. S. ) 2 (1996), 43-91.

[O] A. Ottazzi, Multicontact vector fields on Hessenberg manifolds, J. Lie Theory 15 (2005), 357-377.

[MT] R. MacPherson and J. Tymoczko, "A generalization of Springer's correspondance," in progress.

[dMPS] F. de Mari, C. Procesi, and M. Shayman, "Hessenberg varieties," Trans. Amer. Math. Soc. 332 (1992), 529-534.

[R] K. Rietsch, "Totally positive Toeplitz matrices and quantum cohomology," J. Amer. Math. Soc. 16 (2003), 363-392.

[St] J. Stembridge, "Quasi-miniscule quotients and reduced words for reflections," J. Algebraic Combin. 13 (2001), 275-293.

[T] J. Tymoczko, "Linear conditions imposed on flag varieties," to appear in Amer. J. Math.

Julianna S. Tymoczko

Department of Mathematics, University of Michigan

2074 East Hall, 530 Church Street, Ann Arbor, MI 48109-1043 (734) 936-9932

E-mail: tymoczko@umich.edu 